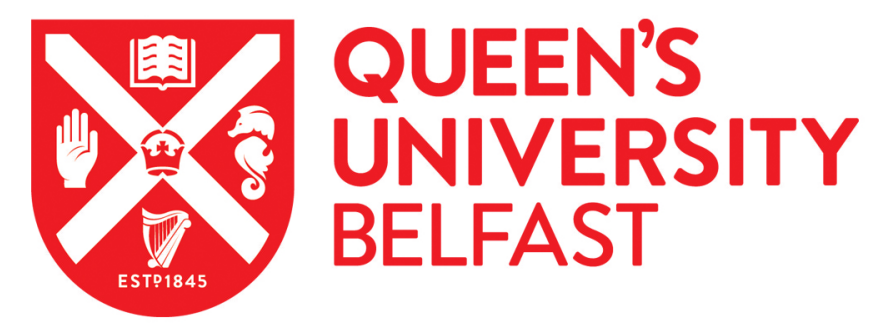

\title{
An Adaptive Backstepping Nonsingular Fast Terminal Sliding Mode Control for Robust Fault Tolerant Control of Robot Manipulators
}

Van, M., Mavrovouniotis, M., \& Ge, S. S. (2019). An Adaptive Backstepping Nonsingular Fast Terminal Sliding Mode Control for Robust Fault Tolerant Control of Robot Manipulators. IEEE Transactions on Systems, Man, and Cybernetics, Part B: Cybernetics, 49(7), 1448-1458. https://doi.org/10.1109/TSMC.2017.2782246

Published in:

IEEE Transactions on Systems, Man, and Cybernetics, Part B: Cybernetics

Document Version:

Peer reviewed version

Queen's University Belfast - Research Portal:

Link to publication record in Queen's University Belfast Research Portal

Publisher rights

(c) 2019 IEEE.

This work is made available online in accordance with the publisher's policies. Please refer to any applicable terms of use of the publisher.

\section{General rights}

Copyright for the publications made accessible via the Queen's University Belfast Research Portal is retained by the author(s) and / or other copyright owners and it is a condition of accessing these publications that users recognise and abide by the legal requirements associated with these rights.

Take down policy

The Research Portal is Queen's institutional repository that provides access to Queen's research output. Every effort has been made to ensure that content in the Research Portal does not infringe any person's rights, or applicable UK laws. If you discover content in the Research Portal that you believe breaches copyright or violates any law, please contact openaccess@qub.ac.uk. 


\title{
An Adaptive Backstepping Nonsingular Fast Terminal Sliding Mode Control for Robust Fault Tolerant Control of Robot Manipulators
}

\author{
Mien Van, Michalis Mavrovouniotis, Member, IEEE, Shuzi Sam Ge, Fellow, IEEE
}

\begin{abstract}
This paper develops a novel control methodology for tracking control of robot manipulators based on a novel adaptive backstepping nonsingular fast terminal sliding mode control (ABNFTSMC). In this approach, a novel backstepping nonsingular fast terminal sliding mode controller (BNFTSMC) is developed based on an integration of integral nonsingular fast terminal sliding mode surface and a backstepping control strategy. The benefits of this approach are that the proposed controller can preserve the merits of the integral nonsingular fast terminal sliding mode control in terms of high robustness, fast transient response, and finite time convergence, and of backstepping control strategy in terms of globally asymptotic stability based on Lyapunov criterion. However, the major limitation of the proposed BNFTSMC is that its design procedure is dependent on the prior knowledge of the bound value of the disturbance and uncertainties. In order to overcome this limitation, an adaptive technique is employed to approximate the upper bound value; yielding an adaptive backstepping nonsingular fast terminal sliding mode control (ABNFTSMC) is recommended. The proposed controller is then applied for tracking control of a PUMA560 robot and compared with other state-of-the-art controllers, such as computed torque controller (CTC), PID controller, conventional PID-based sliding mode controller (PID-SMC), and nonsingular fast terminal sliding mode control (NFTSMC). The comparison results demonstrate the superior performance of the proposed approach.
\end{abstract}

Index Terms-Control of robots, backstepping control, fault tolerant control, terminal sliding mode control.

\section{INTRODUCTION}

$\mathrm{T}$ RACKING CONTROL of robot manipulators, which is required to provide high accuracy, stability and safety in some applications such as industrial robotics, surgical robotics, assistive robotics, in the presence of huge

Manuscript received Aug 10, 2017, accepted Nov 20, 2017. (Corresponding author: Mien Van).

Mien Van is with the Institute of Research and Development, Duy Tan University, Vietnam and the College of Engineering, Mathematics and Physical Sciences, University of Exeter, United Kingdom (e-mail: vanmien1@gmail.com).

Michalis Mavrovouniotis is with the School of Science and Technology, Nottingham Trent University, United Kingdom (e-mail: michalis.mavrovouniotis@ntu.ac.uk).

S. S. Ge is with the Department of Electrical and Computer Engineering and Social Robotics Laboratory, National University of Singapore, 117580 , Singapore (e-mail: samge@nus.edu.sg). uncertainties, disturbance and faults has been a critical issue in both academic and industrial applications [1-3]. How to improve the tracking performance and transient response for robot manipulators, particularly in the presence of external disturbance and possible actuation failures is still a challenge in the research community [4-6].

In order to increase the tracking performance and reliability of the robot, fault tolerant control (FTC) strategies have been developed [7-9]. Generally, FTC approaches can be categorized into two classes: passive FTC (PFTC) and active FTC (AFTC). In AFTC, the control input is adapted according to the fault estimation feedback, which is obtained from a fault diagnosis observer [10-12]. This approach, therefore, requires a design of an additional observer, which will increase the computational burden of the system. In addition, due to the delay of the fault information feedback from the fault diagnosis observer, the fault compensational time of the system is delayed and consequently, the system may become unstable. In contrast, in PFTC approach, one robust controller is designed for both normal and fault operation without requiring any feedback from the fault diagnosis (FD) observer [13-14]. Since the PFTC does not need to wait for the fault information feedback, it can compensate the fault effects much faster. However, since the fault effects imposed on the PFTC system are heavier than that of the active approach, the PFTC requires a control technique with higher robustness.

In the literature, several methodologies have been developed to improve the tracking performance of the robot manipulators in the presence of disturbance, uncertainties and faults. In the initial approaches, PID or PD controller [15-16], computed torque controller (CTC) [6], intelligent and learning control [17-20], optimal control [21-22] and robust control [23-25], etc., have been developed. Among them, robust control techniques have been shown to provide higher robustness and disturbance and fault rejection capability. Sliding mode control (SMC) is a well-known robust control technique and has been widely utilized in real applications due to its strong robustness against uncertainties and disturbance [26]. This great feature of SMC has been applied in the design of FTC systems [2729]. However, the traditional SMC still has four major drawbacks that limit its performance in real applications: 1) it does not provide a finite time convergence, 2) although it possesses a good transient response in normal operation, it is worst to tackle the fast variation effects of the disturbance 
and/or faults, 3) it generates a big chattering in the system, and 4) the design procedure requires a prior knowledge of the upper bound value of the uncertainties, disturbance and faults.

Due to the benefits and limitations of the SMC, many great efforts have been spent on finding effective solutions to preserve the benefits and reduce or eliminate the drawbacks of the conventional SMC. First, in order to obtain a finite time convergence, terminal sliding mode control (TSMC) has been investigated [30-32]. However, the conventional TSMC has a drawback that provides a slow convergence speed and singular problem. In order to tackle the problems of SMC, fast terminal sliding mode control (FTSMC) [33-35] and nonsingular terminal sliding mode control (NTSMC) [36-40] have been developed separately. Unfortunately, the individual approaches based on FTSMC or NTSMC have just only solved one aspect and ignored the other problems of the conventional SMC. In order to obtain both fast finite time convergence and singular elimination, nonsingular fast terminal sliding mode control (NFTSMC) has been proposed [41-43]. Second, in order to enhance the transient response of the conventional SMC, integral sliding mode control (ISMC) or PID-based SMC (PID-SMC) has been developed [44-47]. The basic idea behind this approach is to mimic the property of the integral component in the PID controller to enhance the transient response of the system [48]. In order to obtain both fast transient response and finite time convergence, integral terminal sliding mode control (ITSMC), which combines the benefits of the ISMC and TSMC, have been developed [4950]. However, the conventional ITSMC used the conventional TSMC in the design, and hence the aforementioned weaknesses of the SMC were still present. Third, in order to eliminate the chattering, several approaches have been developed by using either boundary method [51-53] or disturbance observer [54-55] or high-order sliding mode control (HOSMC) [56-60]. Among them, HOSMC is preferred to be used in wide applications since it provides both higher tracking accuracy and chattering elimination. In summary, each drawback of the conventional SMC has been tackled by a corresponding suitable approach, however, there are no approaches in the literature that intended to design a single controller that considers all the drawbacks of SMC together and solve them simultaneously. That is, for the FTC system, the design of SMC needs to guarantee three major demands: fast transient response, finite time convergence and chattering elimination.

Motivated by the above issues, this paper develops a PFTC to tackle the effects of the faults for uncertain robot manipulators using a novel adaptive backstepping nonsingular fast terminal sliding mode control (ABNFTSMC). Starting from the selection of an integral nonsingular fast terminal sliding surface (INFTS), the second-order dynamics model of the robot manipulators based on the position variable is transferred into a third-order dynamics model based on the selected sliding surface. In this way, the control output can be reconstructed such that it can provide a continuous control output like a manner to HOSMC. Since the proposed approach used the integral nonsingular fast terminal sliding mode control and the control output can be reconstructed in a similar manner to the HOSMC, it can preserve the merits of the ITSMC, NFTSMC and HOSMC simultaneously. Therefore the proposed approach will provide a finite time convergence without singular problem, fast transient response, high tracking precision and less chattering. In addition, the proposed design topology follows the design procedure of backstepping control technique [61-64], and thus the globally asymptotic stability of the system can be guaranteed based on the Lyapunov criterion. In summary, the main contributions of this paper are as follows:

- A novel integral nonsingular fast terminal sliding mode surface is proposed.

- A third-order dynamics model of the system is reconstructed based on the selected sliding surface.

- A backstepping design procedure is employed to derive the control output of the third-order dynamics system so that it can achieve a finite time convergence, fast transient response, low steady state error and globally asymptotic stability.

- In comparison with other state-of-the-art methods, such as CTC, PID, PID-SMC and conventional NFTSMC, the proposed approach provides a superior performance.

The remainder of this paper is organized as follows. Section II introduces the problem. Section III presents the robust fault tolerant control based on an adaptive backstepping nonsingular fast terminal sliding mode control (ABNFTSMC). The performance of the controller is verified in Section IV. Section $\mathrm{V}$ provides conclusions.

Remark 1: Beside the references cited and discussed above, there are several other approaches in the literature that were developed for fault tolerant control systems for different applications using different control techniques. For example, in [61-68], FTCs have been established using intelligent learning techniques such as neural network or fuzzy logic. However, the approaches require a huge computational burden due to the weight learning procedure of neural network or fuzzy logic. In [69], an adaptive FTC technique has been developed for uncertain nonlinear time delay systems. In [70], a prescribed performance FTC has been developed. In [71], a FTC scheme has been developed for uncertain linear system over networks with quantization, and so on. However, the focuses of these approaches are out of or far away from the scope of this paper. The focus of this paper is to develop a robust PFTC scheme for uncertain robot manipulators using an advanced robust sliding mode control.

\section{II.PROBLEM STATEMENT}

\section{A. Problem statement}

Consider the robot dynamics described by $[10,42]$ : 


$$
\begin{aligned}
\ddot{q}=M^{-1}(q)(\tau-H(q, \dot{q}) \dot{q}-F(\dot{q}) & \left.-G(q)-\tau_{d}\right) \\
& +\gamma\left(t-T_{f}\right) \phi(q, \dot{q}, \tau)
\end{aligned}
$$

where $q \in \mathfrak{R}^{n}, \dot{q} \in \mathfrak{R}^{n}$ and $\ddot{q} \in \mathfrak{R}^{n} \quad$ denotes the position, velocity and acceleration, respectively. $\tau \in \mathfrak{R}^{n}$ stands for the actuator inputs. $M(q) \in \mathfrak{R}^{n x n}$ is the positive and definite inertia matrix. $H(q, \dot{q}) \in \mathfrak{R}^{n}$ includes the Coriolis and centripetal forces. $F(\dot{q}) \in \mathfrak{R}^{n}$ is the friction matrix. $\tau_{d}$ is a load disturbance matrix and $G(q) \in \mathfrak{R}^{n}$ is the vector of gravity terms. $\phi(q, \dot{q}, \tau) \in \mathfrak{R}^{n} \quad$ represents the fault components affecting on the system, $\gamma\left(t-T_{f}\right) \in \mathfrak{R}^{n}$ represents the time profile of the faults, and $T_{f}$ is the time of occurrence of the faults.

The following standard property is satisfied for the robot dynamics (1):

$0<\lambda_{m}\{M(q)\} \leq\|M\| \leq \lambda_{M}\{M(q)\} \leq \chi, \quad \chi>0$

where $\lambda_{M}\{M\}$ and $\lambda_{m}\{M\}$ are the maximum and minimum eigenvalues of matrix $M$.

The term $\gamma(\cdot)$ is a diagonal matrix having the form below

$$
\gamma\left(t-T_{f}\right)=\operatorname{diag}\left\{\gamma_{1}\left(t-T_{f}\right), \gamma_{2}\left(t-T_{f}\right), \ldots, \gamma_{n}\left(t-T_{f}\right)\right\}
$$

where $\gamma_{i}$ indicates the effects of fault on the $i$ th state equation due to a fault function.

The model of the fault function is introduced by [10, 42]:

$$
\gamma_{i}\left(t-T_{f}\right)= \begin{cases}0 & \text { if } t<T_{f} \\ 1-e^{-a_{i}}\left(t-T_{f}\right) & \text { if } t \geq T_{f}\end{cases}
$$

where $a_{i}>0$ represents the unknown fault evolution rate. The fault is called as incipient fault when the value of $a_{i}$ is small. The fault becomes abrupt fault when the value of $a_{i}$ is large.

Rearranging the dynamics model (1), we have:

$$
\begin{aligned}
\ddot{q}= & M^{-1}(q) \tau+M^{-1}(q)(-H(q, \dot{q}) \dot{q}-G(q)) \\
& +M^{-1}(q)\left(-F(\dot{q})-\tau_{d}\right)+\gamma\left(t-T_{f}\right) \phi(q, \dot{q}, \tau)
\end{aligned}
$$

Define $x_{1}=q$ and $x_{2}=\dot{q}$, the equation (5) can be transformed into a second-order state space model as follows: $\dot{x}_{1}=x_{2}$

$\dot{x}_{2}=M^{-1}\left(x_{1}\right) u+f\left(x_{1}, x_{2}\right)+\Delta$

$y=x_{1}$

where $f\left(x_{1}, x_{2}\right)=M^{-1}(q)(-H(q, \dot{q}) \dot{q}-G(q))$ denotes the lumped known component and $\Delta=M^{-1}(q)\left(-F(\dot{q})-\tau_{d}\right)+\gamma\left(t-T_{f}\right) \phi(q, \dot{q}, \tau)$ denotes the lumped unknown component in the system dynamics.

The objective of this paper is to design a control input $u$ such that the system can provide high tracking performance despite the existence of the uncertainties, disturbance and the presence of faults.

\section{Design of Robust Fault Tolerant Control Based ON ADAPTIVE BACKSTEPPING NONSINGULAR FAST TERMINAL SLIDING MODE CONTROL}

\section{A. Design of robust fault tolerant control based on backstepping nonsingular fast terminal sliding mode control}

In the design of sliding mode control, the selection of the sliding surface has a significant impact on the performance of the system. The sliding surface is chosen such that when it converges to zero, the desired performance of the system can be satisfied. Let $e=x_{1}-x_{d}$ is the trajectory tracking error, where $x_{d}$ denotes a desired trajectory. In order to obtain a fast transient response and finite time convergence without singular problem, an integral nonsingular fast terminal sliding mode (INFTSM) surface is selected as follows:

$$
\sigma_{1}=\int\left(e+k_{1} e^{[\lambda]}+k_{2} e^{[p / q]}\right)
$$

where $\sigma_{1}$ is the sliding variable, $k_{1}=\operatorname{diag}\left(k_{11}, k_{12}, \ldots, k_{1 n}\right) \in \mathfrak{R}^{n \times n}$ and $k_{2}=\operatorname{diag}\left(k_{21}, k_{22}, \ldots, k_{2 n}\right) \in \mathfrak{R}^{n \times n} \quad$ are positive definite matrices, respectively, $p$ and $q$ are positive odd numbers satisfying the relation $1<p / q<2$ and $\lambda>p / q$.

Remark 2: The proposed sliding surface (7) is proposed to combine the properties of the FTSMC [33] and NTSMC [36] such that the system can obtain fast finite time convergence without singular problem. In addition, the integral component is employed for the sliding surface (7) so that the system can possess the merits of the ITSMC, which increases the transient response and reduces the steady state error of the system. When the sliding surface (7) converges to zero, we have: $e+k_{1} e^{[\lambda]}+k_{2} e^{[p / q]}=0$, where $e$ is the terminal attractor of the system. The defined finite time $t_{c}$ that is taken to travel from $e\left(t_{r}\right) \neq 0$ to $e\left(t_{r}+t_{c}\right)=0$ is given by [40]:

$t_{c}=\frac{\frac{p}{q}\left|\mathrm{x}_{1}\left(t_{r}\right)\right|^{1-\frac{q}{p}}}{k_{1}\left(\frac{p}{q}-1\right)} \cdot \Omega\left(\begin{array}{l}\frac{q}{p}, \frac{\frac{p}{q}-1}{(\lambda-1) \frac{p}{q}} ; 1+\frac{\frac{p}{q}-1}{(\lambda-1) \frac{p}{q}} ; \\ -k_{1}\left|\mathrm{x}_{1}\left(t_{r}\right)\right|^{\lambda-1}\end{array}\right)$

where $\Omega$ denotes Gauss' hypergeometric function. The interested readers can refer to [40] for more detail.

The first and second derivative of the sliding surface can be computed as follows:

$\dot{\sigma}_{1}=e+k_{1} e^{[\lambda]}+k_{2} e^{[p / q]}$

$\ddot{\sigma}_{1}=\dot{e}+k_{1} \lambda|e|^{\lambda-1} \cdot \dot{e}+k_{2} \frac{p}{q}|\dot{e}|^{(p / q)-1} \cdot \ddot{e}$

From (6) and (9)-(10), the second-order state space model of the system dynamics based on the state variable can be transformed into a third-order state space model based on the selected sliding surface, as below: 
$\dot{\sigma}_{1}=\sigma_{2}$

$\dot{\sigma}_{2}=\sigma_{3}$

$\dot{\sigma}_{3}=\frac{d}{d t}\left(\begin{array}{l}\dot{e}+k_{1} \lambda|e|^{\lambda-1} \cdot \dot{e}+ \\ k_{2} \frac{p}{q}|\dot{e}|^{(p / q)-1} \cdot\left(M^{-1}\left(x_{1}\right) u+f\left(x_{1}, x_{2}\right)+\Delta-\ddot{x}_{d}\right)\end{array}\right)$

Let $\Psi(e, \dot{e})=\dot{e}+k_{1} \lambda|e|^{\lambda-1} \cdot \dot{e}, \Xi(\dot{e})=k_{2} \frac{p}{q}|\dot{e}|^{(p / q)-1}$, (11) can

be simplified as below:

$\dot{\sigma}_{1}=\sigma_{2}$

$\dot{\sigma}_{2}=\sigma_{3}$

$\dot{\sigma}_{3}=\frac{d}{d t}\left(\Psi(e, \dot{e})+\Xi(\dot{e}) \cdot\left(M^{-1}\left(x_{1}\right) u+f\left(x_{1}, x_{2}\right)+\Delta-\ddot{x}_{d}\right)\right)$

In order to find an effective control input of the system (12), a backstepping design procedure is employed. The following change of coordinates is introduced.

$$
\begin{aligned}
& \vartheta_{1}(t)=\sigma_{1}(t) \\
& \vartheta_{2}(t)=\sigma_{2}(t)-\alpha_{1} \\
& \vartheta_{3}(t)=\sigma_{3}(t)-\alpha_{2}
\end{aligned}
$$

where $\alpha_{i}(i=1,2)$ is the virtual control in the $i$ th step.

\section{Step 1:}

Differentiating $\vartheta_{1}$ in (13) with respect to time, we have

$$
\dot{\vartheta}_{1}=\dot{\sigma}_{1}=\vartheta_{2}+\alpha_{1}
$$

Define a positive definite Lyapunov function $V_{1}=\frac{1}{2} \vartheta_{1}^{2}$. Based on $V_{1}$, virtual control $\alpha_{1}$ can be chosen as $\alpha_{1}(t)=-\xi_{1} \vartheta_{1}(t)$

where $\xi_{1}$ is a positive design parameter. Differentiating $V_{1}$ with respect to time, we have

$$
\begin{aligned}
\dot{V}_{1} & =\vartheta_{1}\left(\vartheta_{2}+\alpha_{1}\right) \\
& =\vartheta_{1}\left(\vartheta_{2}-\xi_{1} \vartheta_{1}\right) \\
& =-\xi_{1}\left|\vartheta_{1}\right|^{2}+\vartheta_{1} \vartheta_{2}
\end{aligned}
$$

From (18), we can see that $\dot{V}_{1}=-\xi_{1}\left|\vartheta_{1}\right|^{2}$ once $\vartheta_{2}=0$, and consequently, $\vartheta_{1}$ will be asymptotically stable.

\section{Step 2:}

Differentiating $\vartheta_{2}$ in (14) with respect to time, we have:

$$
\begin{aligned}
\dot{\vartheta}_{2} & =\dot{\sigma}_{2}-\dot{\alpha}_{1} \\
& =\vartheta_{3}+\alpha_{2}-\dot{\alpha}_{1} \\
& =\vartheta_{3}+\alpha_{2}+\xi_{1} \sigma_{2}
\end{aligned}
$$

Consider the following Lyapunov function:

$V_{2}=V_{1}+\frac{1}{2} \vartheta_{2}^{2}$

Based on (19), the virtual control input is chosen as $\alpha_{2}(t)=-\xi_{2} \vartheta_{2}-\vartheta_{1}-\xi_{1} \sigma_{2}$

Differentiating the Lyapunov function $V_{2}$ with respect to time, we have

$$
\begin{aligned}
\dot{V}_{2} & =\dot{V}_{1}+\vartheta_{2} \dot{\vartheta}_{2} \\
& =-\xi_{1}\left|\vartheta_{1}\right|^{2}+\vartheta_{1} \vartheta_{2}+\vartheta_{2}\left(\vartheta_{3}-\xi_{2} \vartheta_{2}-\vartheta_{1}\right) \\
& =-\xi_{1}\left|\vartheta_{1}\right|^{2}-\xi_{2}\left|\vartheta_{2}\right|^{2}+\vartheta_{2} \vartheta_{3}
\end{aligned}
$$

From (22), $\quad \dot{V}_{2}=-\xi_{1}\left|\vartheta_{1}\right|^{2}-\xi_{2}\left|\vartheta_{2}\right|^{2} \quad$ can be obtained once $\vartheta_{3}=0$ is satisfied. Consequently, the states $\vartheta_{1}$ and $\vartheta_{2}$ will be asymptotically stable.

\section{Step 3:}

Consider the following Lyapunov function

$V_{3}=V_{2}+\frac{1}{2} \vartheta_{3}^{2}$

Differentiating $V_{3}$ with respect to time and combining with the results in (12) and (15), we have:

$\dot{V}_{3}=\dot{V}_{2}+\vartheta_{3} \dot{\vartheta}_{3}$

$$
\begin{aligned}
= & -\xi_{1}\left|\vartheta_{1}\right|^{2}-\xi_{2}\left|\vartheta_{2}\right|^{2}+\vartheta_{2} \vartheta_{3}+ \\
& \vartheta_{3}\left(\frac{d}{d t}\left(\Psi(e, \dot{e})+\Xi(\dot{e}) \cdot\left(\begin{array}{l}
M^{-1}\left(x_{1}\right) u+ \\
f\left(x_{1}, x_{2}\right)+\Delta-\ddot{x}_{d}
\end{array}\right)\right)-\dot{\alpha}_{2}\right)
\end{aligned}
$$

Hence, a backstepping nonsingular fast terminal sliding mode control is designed as

$u(t)=\Xi(\dot{e})^{-1} M\left(x_{1}\right)\left(u_{n}(t)-u_{s}(t)\right)$

where

$$
\begin{aligned}
u_{n}(t)=-\Xi(\dot{e})\left(f\left(x_{1}, x_{2}\right)\right. & \left.-\ddot{x}_{d}\right)-\Psi(e, \dot{e}) \\
& +\alpha_{2}-\int\left(\xi_{3} \vartheta_{3}+\vartheta_{2}\right)
\end{aligned}
$$

and

$$
\dot{u}_{s}(t)=(\Lambda+\zeta) \operatorname{sign}\left(\vartheta_{3}\right)
$$

where $\Upsilon=\frac{d}{d t}(\Xi(\dot{e}) \Delta)$ and $\Lambda$ is selected such that $|\Upsilon| \leq \Lambda . \zeta$ is a positive small constant.

Adding the proposed controller in (25)-(27) into (24), we obtain:

$$
\begin{aligned}
\dot{V}_{3} & =-\xi_{1}\left|\vartheta_{1}\right|^{2}-\xi_{2}\left|\vartheta_{2}\right|^{2}-\xi_{3}\left|\vartheta_{3}\right|^{2}+\vartheta_{3} \frac{d}{d t}\left(u_{s}+\Xi(\dot{e}) \Delta\right) \\
& \leq-\xi_{1}\left|\vartheta_{1}\right|^{2}-\xi_{2}\left|\vartheta_{2}\right|^{2}-\xi_{3}\left|\vartheta_{3}\right|^{2}-(\Lambda+\zeta)\left|\vartheta_{3}\right|+\Upsilon\left|\vartheta_{3}\right| \\
& \leq-\xi_{1}\left|\vartheta_{1}\right|^{2}-\xi_{2}\left|\vartheta_{2}\right|^{2}-\xi_{3}\left|\vartheta_{3}\right|^{2}
\end{aligned}
$$

Then, $\dot{V}_{3} \leq 0, \dot{V}_{3}$ is negative semidefinite, which implies that $\vartheta_{1}(t), \quad \vartheta_{2}(t)$ and $\vartheta_{3}(t)$ will converge to zero in a finite time. Therefore, it can be concluded that the proposed backstepping nonsingular fast terminal sliding mode system is asymptotically stable despite the existing of uncertainties, disturbance and faults.

\section{B. Design of robust fault tolerant control based on adaptive backstepping nonsingular fast terminal sliding mode control}

In the proposed controller (25)-(27), the design procedure is based on the assumption that the upper bound value $\Lambda$ of the unknown function $\Upsilon$ can be obtained in advance. However, this assumption is too conservative in practical engineering applications since it is difficult to estimate the bound value of 
the uncertainty, disturbance and fault parameters in advance. In order to resolve this limitation, a simple adaptive law is developed in this subsection, and an adaptive backstepping nonsingular fast terminal sliding mode control (ABNFTSMC) is proposed as a result.

Steps $\mathbf{1}$ and $\mathbf{2}$ of the ABNFTSMC are designed as the same procedure as the BNFTSMC. However, in Step 3, an adaptive control law $u_{a s}$ is used to replace the control law $u_{s}$ in (27), which requires an upper bound value of the disturbance and faults. That is:

$$
u(t)=\Xi(\dot{e})^{-1} M\left(x_{1}\right)\left(u_{n}(t)-u_{a s}(t)\right)
$$

where $u_{n}(t)$ is designed just like in (26), and the adaptive reaching law can be designed as

$\dot{u}_{a s}(t)=(\hat{\Lambda}+\zeta) \operatorname{sign}\left(\vartheta_{3}\right)$

where $\hat{\Lambda}$ is used to approximate the upper bound value $\Lambda$. It is adapted using the following law:

$\dot{\hat{\Lambda}}=\frac{1}{\delta}\left|\vartheta_{3}\right|$

where $\delta$ is a user defined constant.

Let $\tilde{\Lambda}=\Lambda-\hat{\Lambda}$ is the estimation error. We consider the following Lyapunov function for Step 3:

$V_{4}=V_{3}+\frac{1}{2} \delta \tilde{\Lambda}^{2}$

In combination with the result in (24), the derivative of the Lyapunov function (32) can be obtained as

$$
\begin{aligned}
\dot{V}_{4}= & \dot{V}_{3}+\delta(\hat{\Lambda}-\Lambda) \dot{\hat{\Lambda}} \\
= & -\xi_{1}\left|\vartheta_{1}\right|^{2}-\xi_{2}\left|\vartheta_{2}\right|^{2}+\vartheta_{2} \vartheta_{3}+\delta(\hat{\Lambda}-\Lambda) \dot{\hat{\Lambda}} \\
& \vartheta_{3}\left(\frac{d}{d t}\left(\Psi(e, \dot{e})+\Xi(\dot{e}) \cdot\left(\begin{array}{l}
M^{-1}\left(x_{1}\right) u+ \\
f\left(x_{1}, x_{2}\right)+\Delta-\ddot{x}_{d}
\end{array}\right)\right)-\dot{\alpha}_{2}\right)
\end{aligned}
$$

Adding the controller (29)-(31) into (33), we obtain

$$
\begin{aligned}
\dot{V}_{4}= & -\xi_{1}\left|\vartheta_{1}\right|^{2}-\xi_{2}\left|\vartheta_{2}\right|^{2}-\xi_{3}\left|\vartheta_{3}\right|^{2} \\
& +\vartheta_{3} \frac{d}{d t}\left(u_{a s}+\Xi(\dot{e}) \Delta\right)+\delta(\hat{\Lambda}-\Lambda) \dot{\hat{\Lambda}} \\
= & -\xi_{1}\left|\vartheta_{1}\right|^{2}-\xi_{2}\left|\vartheta_{2}\right|^{2}-\xi_{3}\left|\vartheta_{3}\right|^{2} \\
& +\vartheta_{3}\left(-(\hat{\Lambda}+\zeta) \operatorname{sign}\left(\vartheta_{3}\right)+\frac{d}{d t}(\Xi(\dot{e}) \Delta)\right)+\delta(\hat{\Lambda}-\Lambda) \dot{\hat{\Lambda}} \\
\leq & -\xi_{1}\left|\vartheta_{1}\right|^{2}-\xi_{2}\left|\vartheta_{2}\right|^{2}-\xi_{3}\left|\vartheta_{3}\right|^{2}-(\Lambda+\zeta)\left|\vartheta_{3}\right|+\Upsilon\left|\vartheta_{3}\right| \\
\leq & -\xi_{1}\left|\vartheta_{1}\right|^{2}-\xi_{2}\left|\vartheta_{2}\right|^{2}-\xi_{3}\left|\vartheta_{3}\right|^{2}
\end{aligned}
$$

Then, $\dot{V}_{4} \leq 0, \dot{V}_{4}$ is negative semidefinite, which implies that $\vartheta_{1}(t), \quad \vartheta_{2}(t)$ and $\vartheta_{3}(t)$ will converge to zero in a finite time. Therefore, it can be concluded that the proposed backstepping nonsingular fast terminal sliding mode system is asymptotically stable despite the existing of uncertainties, disturbance and faults.

Remark 3: In order to eliminate the parameter drift problem when implementing the adaptive law (31), the following deadzone technique is employed:

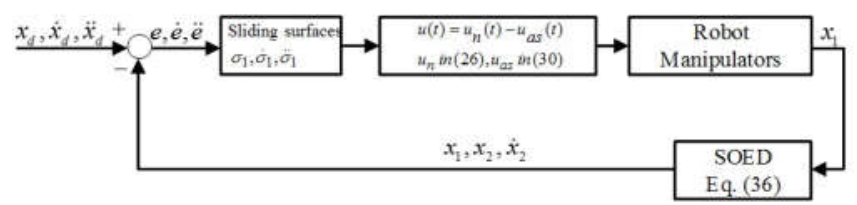

Fig. 1. Illustration of the control structure of the system.

$\dot{\hat{\Lambda}}= \begin{cases}0 & \text { if }\left|\vartheta_{3}\right| \leq \varepsilon \\ \frac{1}{\delta}\left|\vartheta_{3}\right| & \text { if }\left|\vartheta_{3}\right|>\varepsilon\end{cases}$

where $\varepsilon$ is the dead zone size.

Remark 4: The design of the proposed controller requires the measurements of position, velocity and acceleration. However, in practical robot, only position is measureable based on the encoder information. To estimate the velocity and acceleration measurement, the second-order exact differentiation (SOED) [57] is employed in this paper:

$$
\begin{aligned}
& \dot{\omega}_{0}=\kappa_{0} \\
& \kappa_{0}=-m_{1}\left|\omega_{0}-x_{1}\right|^{2 / 3} \operatorname{sign}\left(\omega_{0}-x_{1}\right)+\omega_{1} \\
& \dot{\omega}_{1}=\kappa_{1} \\
& \kappa_{1}=-m_{2}\left|\omega_{1}-\kappa_{0}\right|^{1 / 2} \operatorname{sign}\left(\omega_{1}-\kappa_{0}\right)+\omega_{2} \\
& \dot{\omega}_{2}=-m_{3} \operatorname{sign}\left(\omega_{2}-\kappa_{1}\right) .
\end{aligned}
$$

Then, the estimates of the position, velocity and acceleration can be obtained as

$\omega_{0}=x_{1}, \omega_{1}=x_{2}, \omega_{2}=\dot{x}_{2}$

Remark 5: The design procedure of the proposed controller is based on the assumption that the derivative of the unknown component $\Upsilon$ are bounded by an unknown constant. However, this assumption may not be obtained in some conditions when the change of fault are fast, and thus the system will be unstable. Fortunately, due to the faster transient response of the proposed control method, which combines the backstepping and integral nonsingular fast terminal sliding mode control, the unstable condition may occur only for a very short time and the system will be stable quickly after then. This symptom will be illustrated in section IV.

Remark 6: The approximation learning techniques based on neural network and fuzzy logic can be used to approximate the unknown component $\Upsilon$ as a similar design procedure to [5966]. However, the design of approximation techniques based on neural network or fuzzy logic are computational expensive.

\section{RESUlTS AND DisCUSSIONS}

In order to verify the performance of the prosed approach, we employ it for a PUMA560 robot [72]. For simplicity in analyzing and presenting the results, only the first three joints of the robot are used. The dynamic and kinematic model of the PUMA560 robot are described as in [72].

The friction and disturbance term are modeled as

$$
F(q)+\tau_{d}=\left[\begin{array}{l}
0.5 \dot{q}_{1}+\sin \left(3 q_{1}\right)+0.5 \sin \left(\dot{q}_{1}\right) \\
1.3 \dot{q}_{2}-1.8 \sin \left(2 q_{2}\right)+1.1 \sin \left(\dot{q}_{2}\right) \\
-1.8 \dot{q}_{3}+2 \sin \left(3 q_{3}\right)+0.15 \sin \left(\dot{q}_{3}\right)
\end{array}\right]
$$


TABLE I

SELECTED PARAMETERS OF THE CONTROLLERS

\begin{tabular}{lll}
\hline Controller & Parameters & Value \\
\hline CTC & $K_{p}, K_{d}$ & 200,10 \\
PID & $K_{p}, K_{i}, K_{d}$ & $200,100,10$ \\
PID-SMC & $K_{p}, K_{i}, K_{d}$ & $200,100,10$ \\
& $\bar{\Delta}, \zeta, v$ & $20,1,0.1$ \\
NFTSMC & $k_{1}, k_{2}, \lambda, p, q$ & $10,5,1.4,9,7$ \\
& $\bar{\Delta}, \zeta, v$ & $20,1,0.1$ \\
ABNFTSMC & $k_{1}, k_{2}, \lambda, p, q$ & $10,5,1.4,9,7$ \\
& $\xi_{1}, \xi_{2}, \xi_{3}$ & $200,100,10$ \\
& $\delta, \varepsilon$ & $0.5,0.01$ \\
\hline
\end{tabular}

TABLE II

Tracking ERRORS AN ELAPSED TIME (ET) OF THE SYSTEM UNDER THE INPUT OF THE CONTROLLERS

\begin{tabular}{lllll}
\hline $\begin{array}{c}\text { Error } \\
\text { Controller }\end{array}$ & $E_{1}$ & $E_{2}$ & $E_{3}$ & ET(s) \\
\hline CTC & 0.4580 & 0.5938 & 0.2696 & 2.50 \\
PID & 0.2417 & 0.3698 & 0.1768 & 2.04 \\
PID-SMC & 0.0593 & 0.0478 & 0.0472 & 3.83 \\
NFTSMC & 0.1119 & 0.2453 & 0.0768 & 4.07 \\
ABNFTSMC & 0.0248 & 0.0219 & 0.0118 & 4.85 \\
\hline
\end{tabular}

The desired trajectory of the system is selected as follows:

$$
x_{d}=\left[\cos (t / 5 \pi)-1, \cos \left(t / 5 \pi+\frac{\pi}{2}\right), \sin \left(t / 5 \pi+\frac{\pi}{2}\right)-1\right]^{T}
$$

The Matlab/Simulink environment using Runge-Kutta algorithm are used to conduct experiments. The sampling time is set as $0.005 \mathrm{~s}$.

In this paper, we consider the performance of the proposed method in both normal and fault operations. In order to demonstrate the superior performance of the proposed ABNFTSMC controller, we compare it with the state-of-theart controllers, which have been proposed for improving trajectory tracking performance of robot manipulators such as computed torque control (CTC) [6], PID controller [15-16], conventional PID-based SMC controller (PID-SMC) [48], and conventional nonsingular fast terminal sliding mode control (NFTSMC) [41- 43]. The design of the CTC, PID, PID-based SMC (PID-SMC) and NFTSMC are represented in Appendix $\mathrm{A}, \mathrm{B}, \mathrm{C}$ and $\mathrm{D}$, respectively. The selected parameters of these controllers are reported in TABLE I.

First, we assume that the system is operated in normal working condition with the assumed uncertainties and disturbance. The tracking performance of the system in Cartesian space under the controllers are shown in Fig. 2. For an easier comparison, the joint tracking errors $\left(e=x_{1}-x_{d}\right)$ of the system are shown in Fig. 3. From Figs. 2 and 3, we can see that the CTC provides worst tracking performance for the system in case of the presence of the disturbance and uncertainties. The PID controller provides better tracking

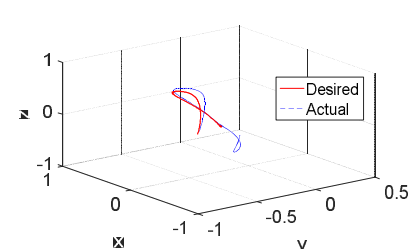

(a)

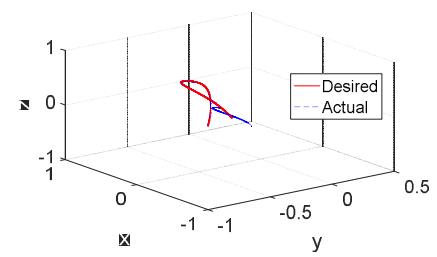

(c)

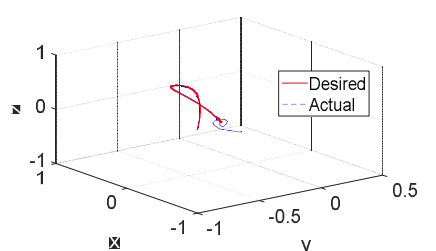

(b)

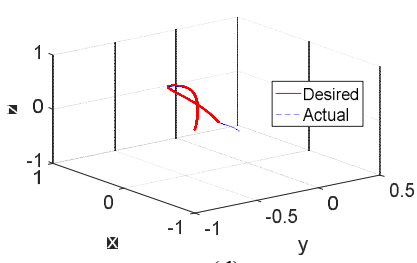

(d)

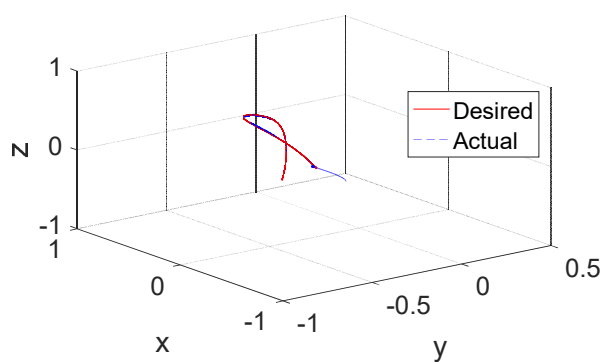

(e)

Fig. 2. Desired position trajectory and actual position trajectory of the system under (a) CTC, (b) PID, (c) PID-based SMC, (d) NFTSMC and (e) ABNFTSMC controllers.

performance compared to the CTC. However, the convergence times and steady state errors of these controllers are very large. Due to the robustness property of the SMC against the effects of the disturbance and uncertainties, the PID-SMC, NFTSMC and ABNFTSMC provide better performance compared to the CTC and PID controllers. Interestingly, due to the benefits of the integral component in the design, the PID-SMC and ABNFTSMC provide lower steady state error and faster transient response than the NFTSMC. In addition, the ABNFTSMC provides the best performance since it possesses low steady state error and fast transient response properties of the NFTSMC and ITSMC.

Then, we consider the fault tolerant capability of these controllers. To model the effects of faults in the system, we assume that the following fault function exits in the system:

$$
\phi(q, \dot{q}, \tau)=\left[\begin{array}{ll}
\left(\begin{array}{ll}
30 \sin \left(q_{1} q_{2}\right)+4 \cos \left(\dot{q}_{1} q_{2}\right) \\
+15 \cos \left(\dot{q}_{1} \dot{q}_{2}\right)
\end{array}\right) & \text { with } \mathrm{T}_{f 1} \geq 20 \\
-0.8 u_{2} & \text { with } \mathrm{T}_{f 2} \geq 30 \\
0 &
\end{array}\right]
$$

It means that we assume an abrupt fault $\phi_{1}=30 \sin \left(q_{1} q_{2}\right)+4 \cos \left(\dot{q}_{1} q_{2}\right)+15 \cos \left(\dot{q}_{1} \dot{q}_{2}\right) \quad$ occurs in the first actuator from the time $t \geq 20 \mathrm{~s}$, while the second actuator losses $80 \%$ its effectiveness from the time $t \geq 30 \mathrm{~s}$. The third actuator is assumed to be healthy. The tracking errors of the system under the controllers are reported in Fig. 4. For an easier comparison, the averaged tracking error defined in (41) 

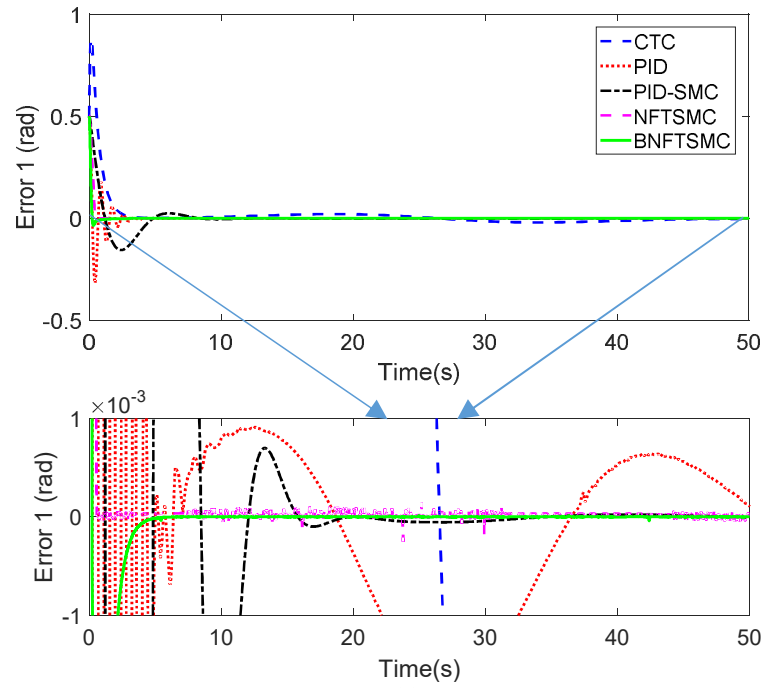

(a)
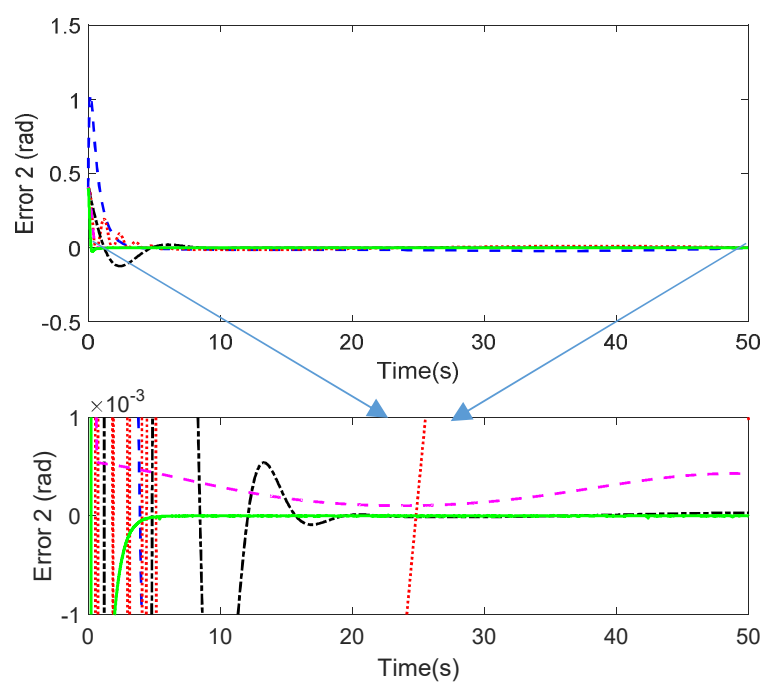

(b)
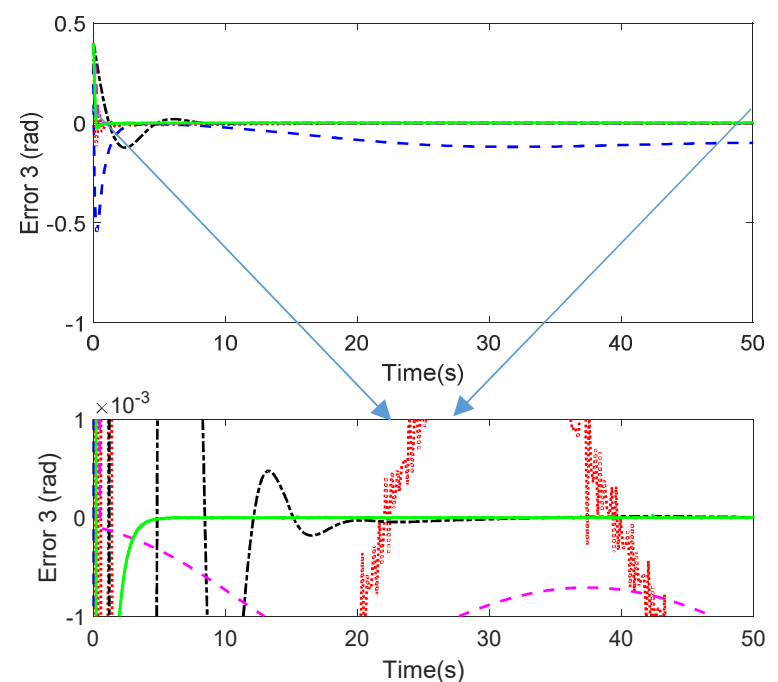

(c)

Fig. 3. Joint trajectory tracking error when the system is in normal operation (a) Joint 1, (b) Joint 2, and (c) Joint 3.
$E_{j}=\sqrt{\frac{1}{N} \sum_{i=1}^{N}\left(\left\|e_{j}(k)\right\|^{2}\right)} j=1,2,3$

where $N$ is the number of simulation step, and the elapsed time (ET) defined in Remark 7 are also reported in TABLE II.

From the results shown in Fig. 4 and TABLE II, we can see that the CTC and PID provide worst tracking performances for the system in the presence of fault; the system becomes unstable immediately when the faults occur. Even though the NFTSMC provides better tracking performance, the system is easily to be unstable due to the effects of faults, as shown in Fig. 4b). In the presence of faults, the benefits of the integral component are crucially significant, as shown in the performance of the PID-SMC and ABNFTSMC; using the PID-SMC and ABNFTSMC, the system provides very fast transient response and low tracking error. In addition, as shown in Fig. 4, the proposed ABNFTMC provides superior performance compared to the PID-SMC. However, as shown in TABLE II, the elapsed time (ET) of the PID (2.04), CTC (2.50) and PID-SMC (3.83) is smaller compared to the NFTSMC (4.07) and ABNFTSMC (4.85). Fortunately, compared to the elapsed time of the PID, which is known as the simplest control method, the computational time of the proposed method is not significantly higher. Therefore, with the current advance hardware technologies, it is possible to apply the proposed technique in practical applications.

The control efforts of the NFTSMC, PID-SMC and ABNFTSMC are shown in Fig. 5. From Fig. 5(c), we can see that the proposed ABNFTSMC provides a continuous control input. As shown in Fig. 5(a) and (b), the PID-SMC and NFTSMC controllers also provide continuous control input due to the used boundary function (49). However, these approaches, as mentioned above, reduce the robustness and increase the steady state error of the system. The variation of the adaptive gains of the proposed ABNFTSMC for normal and fault operations are shown in Figs. 6 and 7, respectively. From the Figs. 6 and 7 we can see that the adaptive gains are variant due to the change of the effects of the uncertainties, disturbance and faults, and they will converge to some stable values when the systems are stable. In addition, the comparison results between Fig. 6 and Fig. 7 show that the adapted gains do not change much when the faults occur; the gains are just slightly increased when the faults occur. This is because the fault effects have been compensated by the gain component of the backstepping control $\left(\alpha_{2}-\int\left(\xi_{3} \vartheta_{3}+\vartheta_{2}\right)\right)$ in the design. This is also one of the major merits of the proposed approach.

From the above results we can conclude that the proposed controller, ABNFTSMC, provides the best performance compared to other controllers such as CTC, PID, SMC-PID and NFTSMC in terms of tracking accuracy, transient response, chattering elimination, and low steady state error.

Remark 7: The elapsed time shown in TABLE II in this paper is the time that the Matlab has used to complete 50second simulation time. This may not reflect the true computational burden of the controllers but it can be used to provide a general idea about the comparison in computational time among the controllers. 
Remark 8: From the developed control law, i.e., ABNFTSMC, in (26) and (29-31) and the selected control parameters in TABLE I, we can see that the system's performance is dependent on the selection of the sliding surface parameters $k_{1}, k_{2}, \lambda, p, q$, the adaptive gain parameters $\delta, \varepsilon$ and the backstepping gains $\xi_{1}, \xi_{2}, \xi_{3}$. While the sliding surface parameters and the adaptive gain parameters have been discussed widely in the literature, the backstepping gains are new defined parameters in this paper and their effects on the system performance are significant. Therefore, these parameters need to be effectively selected. In this paper, the backsteeping gain parameters are chosen based on experiments. However, the values chosen based on the experiments are usually not optimal. Hence, there is a need to investigate a tuning mechanism to tune the parameters such that the system can get good approximation response. This will be studied in our future works.

Remark 9: From the proof for the stability of the system described in Section III, the stability of the system can be guaranteed when the sliding gain is selected such that its value is bigger than the value $\Lambda$, which is the bound value of the unknown function $\Upsilon$. Therefore, the stability of the system can be guaranteed when the adaptive gain is adapted to be bigger than the value $\Lambda$. In Figs. (6) and (7) we can see that the adaptive sliding gains of joints 2 and 3 converge after some transient time. The sliding gain of joint 1 is increased very slightly after some transient time. This symptom is caused due to the parameter drift problem as discussed in Remark 2. The convergence speed of the adaptive parameters can be obtained faster if we increase the dead zone size $\varepsilon$. However, there is a tradeoff between the steady state error and the convergence speed of the system when the dead zone size $\varepsilon$ is changed. When the value of $\varepsilon$ is high, the convergence speed is fast but the steady state error is high, and vice versa.

Remark 10: In this simulation study, we simulate the effects of abrupt faults only since its effects in the system are heavier than the incipient faults. Since the proposed method can compensate the abrupt faults well, it would absolutely be able to tackle the effects of the incipient faults well.

Remark 11: In this paper, we design a robust fault tolerant control to compensate for the effects of actuator or component faults only. The proposed method may not be able to compensate for the sensor faults. The effects of the measurement noise and the sensor faults to the performance of the system will be studied in our future work.

Remark 12: From the viewpoint of control engineering, it would be good to use experimental results to verify the effectiveness of the proposed control scheme in practical applications. However, simulation of different fault effects in the practical experiments are difficult, dangerous and possibly damage the system. Therefore, almost every approaches for fault tolerant control systems in the literatures [59-62] and the work in this paper used the simulation environment to verify the effectiveness of the methods. However, verifying the proposed approach in practical experiments by using effective manners without damaging the system and the environment surrounds is necessary and this will be investigated in our future works.
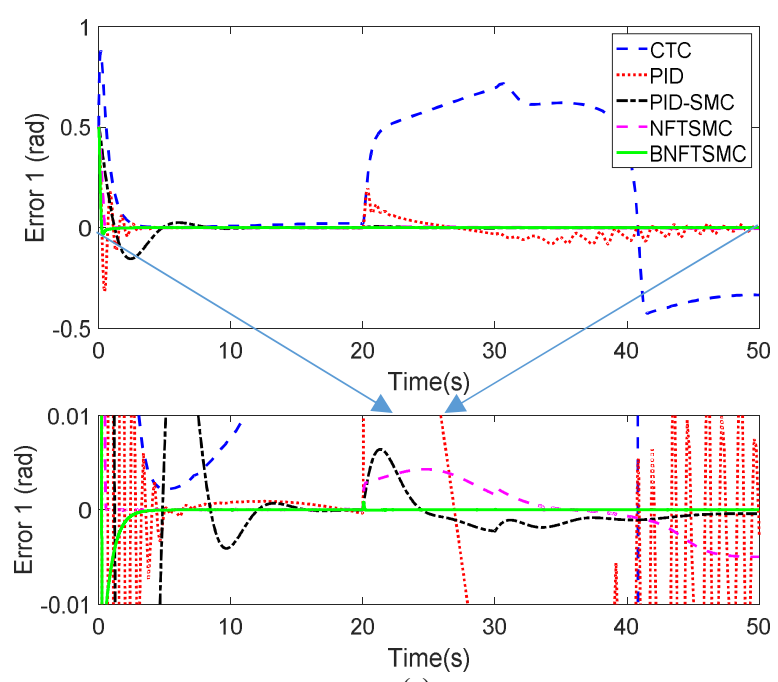

(a)
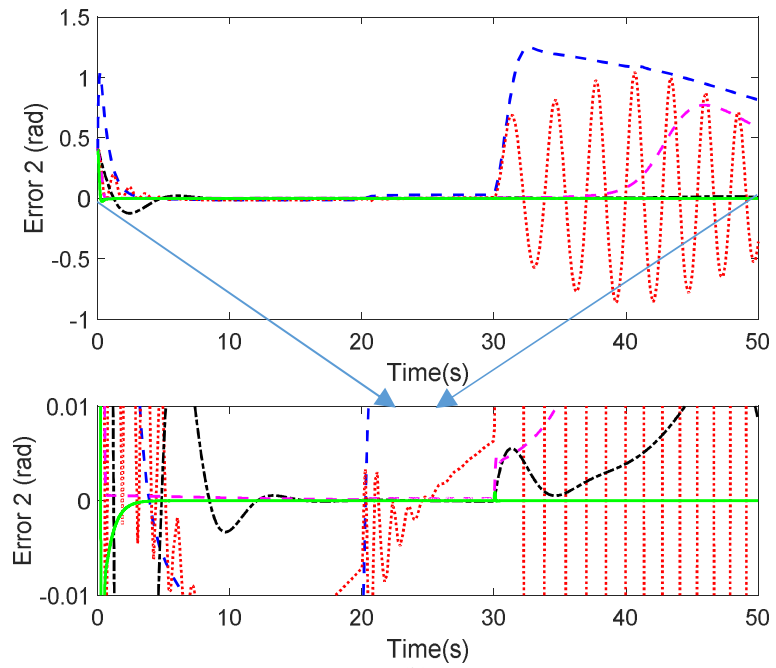

(b)
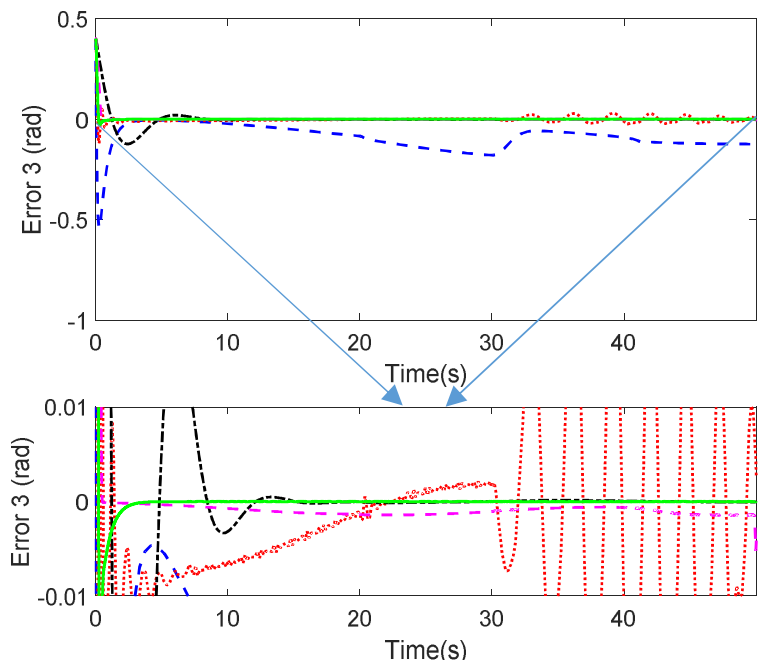

(c)

Fig. 4. Joint trajectory tracking error when the system is in fault operation. (a) Joint 1, (b) Joint 2, and (c) Joint 3. 


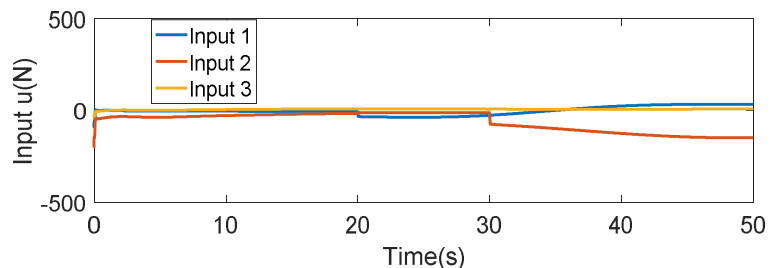

(a)

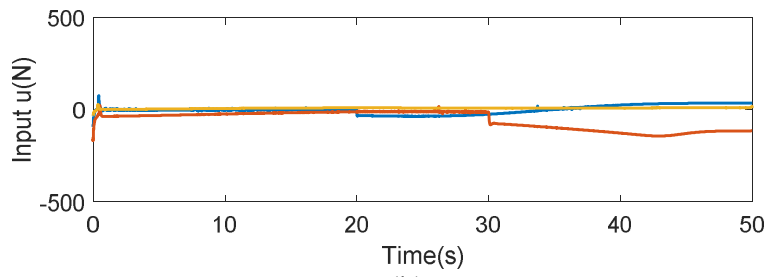

(b)

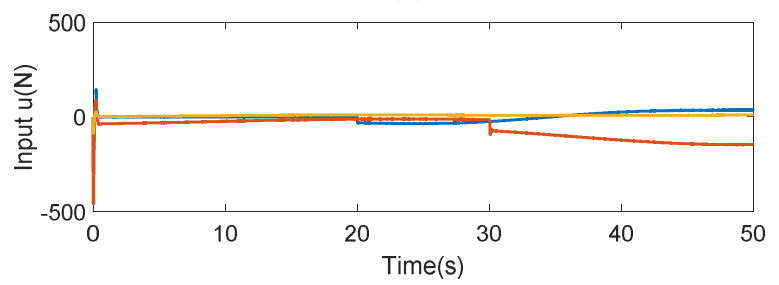

(c)

Fig. 5. Control input of the system under: (a) PID-SMC, (b) NFTSMC, and (c) ABNFTSMC.

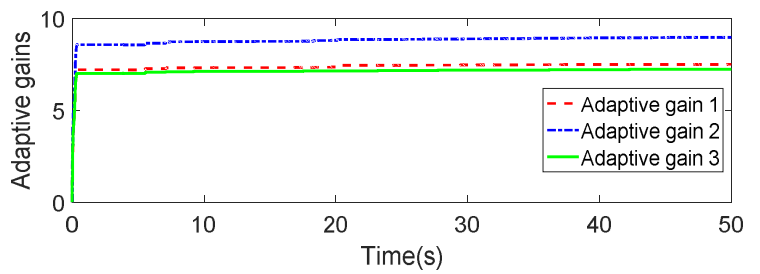

Fig. 6. Variation of the adaptive gains in (35) of the proposed ABNFTSMC when the system is in normal operation.

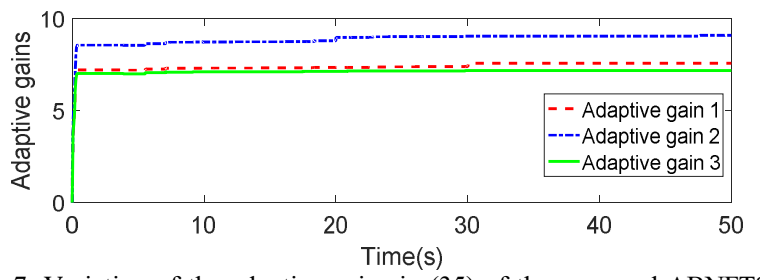

Fig. 7. Variation of the adaptive gains in (35) of the proposed ABNFTSMC when the system is in fault operation.

\section{V.CONCLUSIONS}

This paper develops a novel robust tracking control algorithm for robot manipulators with an enhancing on uncertainties and disturbance rejection and faults compensation capability. The proposed approach combines a novel integral nonsingular fast terminal sliding mode control (INFTSMC) and a backstepping design mechanism. The outstanding feature of the developed INFTSMC is that it can integrate the benefits of the integral sliding mode control, nonsingular fast terminal sliding mode control and high order sliding mode control using an effective way so that a single controller could provide many great features such as fast transient response, low tracking error and low chattering. In addition, the proposed controller is designed based on the backstepping control procedure, and thus it can provide globally asymptotic stability based on Lyapunov criteria. The proposed controller is then applied for a PUMA560 robot and compared with other state-of-the-art controllers such as CTC, PID, PID-SMC and NFTSMC. The simulation results verify the superior performance of the proposed method in terms of uncertainties and disturbance rejection in normal operation and fault compensation in fault operation.

For future work, the effects of the measurement noises and sensor faults to the system control performance will be studied. Tuning mechanisms will also be developed to obtain the optimal values for the major parameters of the proposed controller.

\section{APPENDIX A}

The following is the design procedure of the computed torque controller:

The computed torque controller can be designed as

$u=M\left(x_{1}\right)\left(\ddot{x}_{d}+K_{p}\left(x_{d}-x_{1}\right)+K_{d}\left(\dot{x}_{d}-x_{2}\right)\right)+f\left(x_{1}, x_{2}\right)$

where $K_{p}$ is the proportional gain matrix and $K_{d}$ is the derivative gain matrix.

\section{APPENDIX B}

The following is the design procedure of the PID controller: Let $e=x_{1}-x_{d}$ is the tracking error. Then, the PID controller law is designed as

$u=K_{p} e+K_{d} \dot{e}+K_{i} \int_{0}^{t} e(t) d t$

where $K_{p}$ is the proportional gain matrix, $K_{d}$ is the derivative gain matrix and $K_{i}$ is the integral gain matrix.

\section{APPENDIX C}

The following is the design procedure of the PID-based SMC (PID-SMC) controller:

Let $e=x_{1}-x_{d}$ is the tracking error. Then, the sliding surface is selected as

$\sigma=K_{p} e+K_{d} \dot{e}+K_{i} \int_{o}^{t} e(t) d t$

where $K_{p}$ is the proportional gain matrix, $K_{d}$ is the derivative gain matrix and $K_{i}$ is the integral gain matrix.

The derivative of the sliding surface is obtained as

$$
\begin{aligned}
\dot{\sigma} & =K_{p} \dot{e}+K_{d} \ddot{e}+K_{i} e \\
& =K_{p} \dot{e}+K_{d}\left(M^{-1}\left(x_{1}\right) u+f\left(x_{1}, x_{2}\right)+\Delta-\ddot{x}_{d}\right)+K_{i} e
\end{aligned}
$$

From (45), the PID-SMC is selected as 
$u(t)=u_{e q}(t)-u_{s}(t)$

where, the equivalent control law is reconstructed when $\dot{\sigma}=0$ and $\Delta=0$ :

$u_{e q}=M\left(x_{1}\right)\left(-\frac{K_{i}}{K_{d}} e-\frac{K_{p}}{K_{d}} \dot{e}-\left(f\left(x_{1}, x_{2}\right)-\ddot{x}_{d}\right)\right)$

and, the reaching law is designed as

$u_{s}=M\left(x_{1}\right)(\bar{\Delta}+\zeta) \operatorname{sign}(\sigma)$

where $\bar{\Delta}$ is chosen such that the unknown component is bounded by $\|\Delta\| \leq \bar{\Delta}$.

In order to alleviate the chattering phenomenon due to the use of $\operatorname{sign}(s)$ function. The continuous function below is used instead:

$u_{s}(t)=M\left(x_{1}\right)(\bar{\Delta}+\zeta) \frac{s}{\|s\|+v}$

where $v \in \mathfrak{R}$ is a small positive scalar.

\section{APPENDIX D}

The following is the design procedure of the nonsingular fast terminal sliding mode controller (NFTSMC).

Let $e=x_{1}-x_{d}$ is the tracking error. Then, the NFTSMC sliding surface is selected as

$\sigma=\mathrm{e}+k_{1} \mathrm{e}^{[\lambda]}+k_{2} \mathrm{e}^{[p / q]}$

where $\sigma$ is the sliding variable,

$k_{1}=\operatorname{diag}\left(k_{11}, k_{12}, \ldots, k_{1 n}\right) \in \mathfrak{R}^{n \times n}$

$k_{2}=\operatorname{diag}\left(k_{21}, k_{22}, \ldots, k_{2 n}\right) \in \mathfrak{R}^{n \times n} \quad$ are positive definite matrices, respectively, $p$ and $q$ are positive odd numbers satisfying the relation $1<p / q<2$ and $\lambda>p / q$.

The first derivative of the sliding surface can be computed as follows:

$$
\begin{aligned}
\dot{\sigma}= & \dot{e}+k_{1} \lambda|e|^{\lambda-1} \cdot \dot{e} \\
& +k_{2} \frac{p}{q}|\dot{e}|^{(p / q)-1} \cdot\left(\begin{array}{l}
M^{-1}\left(x_{1}\right) u \\
+f\left(x_{1}, x_{2}\right)+\Delta-\ddot{x}_{d}
\end{array}\right)
\end{aligned}
$$

Based on (51), the NFTSMC controller is designed as $u(t)=u_{e q}(t)-u_{s}(t)$

where, the equivalent control law is reconstructed when $\dot{\sigma}=0$ and $\Delta=0$ :

$u_{e q}=M\left(x_{1}\right)\left(\begin{array}{l}-\frac{1}{k_{2}} \frac{q}{p} e\left(\dot{e}^{[2-p / q]}+k_{1} \lambda|e|^{\lambda-1} \dot{e}^{[2-p / q]}\right) \\ -f\left(x_{1}, x_{2}\right)+\ddot{x}_{d}\end{array}\right)$

and the reaching law is designed as

$u_{s}=M\left(x_{1}\right)(\bar{\Delta}+\zeta) \operatorname{sign}(\sigma)$
In order to reduce the chattering, the boundary method in (49) is also used to replace the (54).

Remark 13: The stability proofs for the controllers in the Appendixes are omitted to reduce the length of the paper.

\section{REFERENCES}

[1] H. Yu, J. H. Shen, K. M. Joos and N. Simaan, "Calibration and Integration of B-Mode Optical Coherence Tomography for Assistive Control in Robotic Microsurgery," IEEE/ASME Transactions on Mechatronics, vol. 21, no. 6, pp. 2613-2623, Dec. 2016.

[2] F. Lunardini, C. Casellato, A. d'Avella, T. D. Sanger and A. Pedrocchi, "Robustness and Reliability of Synergy-Based Myocontrol of a Multiple Degree of Freedom Robotic Arm," IEEE Transactions on Neural Systems and Rehabilitation Engineering, vol. 24, no. 9, pp. 940-950, Sept. 2016.

[3] K. C. Lau, E. Y. Y. Leung, P. W. Y. Chiu, Y. Yam, J. Y. W. Lau and C. C. Y. Poon, "A Flexible Surgical Robotic System for Removal of EarlyStage Gastrointestinal Cancers by Endoscopic Submucosal Dissection," IEEE Transactions on Industrial Informatics, vol. 12, no. 6, pp. 23652374, Dec. 2016.

[4] M. Van; S. S. Ge; D. Ceglarek, "Fault Estimation and Accommodation for Virtual Sensor Bias Fault in Image-Based Visual Servoing using Particle Filter," IEEE Transactions on Industrial Informatics, vol.PP, no.99, doi: 10.1109/TII.2017.2723930

[5] M. Van, D. Wu, S. S. Ge and H. Ren, "Fault Diagnosis in Image-Based Visual Servoing With Eye-in-Hand Configurations Using Kalman Filter," IEEE Transactions on Industrial Informatics, vol. 12, no. 6, pp. 1998-2007, Dec. 2016.

[6] M. Van, H. J. Kang, Y. S. Suh,“ A novel neural second-order sliding mode observer for robust fault diagnosis in robot manipulators," International Journal of Precision Engineering and Manufacturing, vol. 14, no. 3, pp. 397-406, 2013.

[7] G. Chen, Y. Song and F. L. Lewis, "Distributed Fault-Tolerant Control of Networked Uncertain Euler-Lagrange Systems Under Actuator Faults," IEEE Transactions on Cybernetics, vol. 47, no. 7, pp. 17061718, July 2017.

[8] K. M. Ben-Gharbia, A. A. Maciejewski and R. G. Roberts, "A Kinematic Analysis and Evaluation of Planar Robots Designed From Optimally Fault-Tolerant Jacobians," IEEE Transactions on Robotics, vol. 30, no. 2, pp. 516-524, April 2014.

[9] R. C. Hoover, R. G. Roberts, A. A. Maciejewski, P. S. Naik and K. M. Ben-Gharbia, "Designing a Failure-Tolerant Workspace for Kinematically Redundant Robots," IEEE Transactions on Automation Science and Engineering, vol. 12, no. 4, pp. 1421-1432, Oct. 2015.

[10] M. Van, H. J. Kang, "A robust fault diagnosis and accommodation scheme for robot manipulators," International Journal of Control, Automation and Systems, vol. 11, no. 2, pp. 377-388, 2013.

[11] X. He, Z. Wang, L. Qin and D. Zhou, "Active Fault-Tolerant Control for an Internet-Based Networked Three-Tank System," IEEE Transactions on Control Systems Technology, vol. 24, no. 6, pp. 2150-2157, Nov. 2016.

[12] Q. Shen, B. Jiang and V. Cocquempot, "Adaptive Fuzzy ObserverBased Active Fault-Tolerant Dynamic Surface Control for a Class of Nonlinear Systems With Actuator Faults," IEEE Transactions on Fuzzy Systems, vol. 22, no. 2, pp. 338-349, April 2014

[13] M. Benosman and K. Y. Lum, "Passive Actuators' Fault-Tolerant Control for Affine Nonlinear Systems," IEEE Transactions on Control Systems Technology, vol. 18, no. 1, pp. 152-163, Jan. 2010.

[14] R. Wang and J. Wang, "Passive Actuator Fault-Tolerant Control for a Class of Overactuated Nonlinear Systems and Applications to Electric Vehicles," IEEE Transactions on Vehicular Technology, vol. 62, no. 3, pp. 972-985, March 2013.

[15] W. Yu and J. Rosen, "Neural PID Control of Robot Manipulators With Application to an Upper Limb Exoskeleton," IEEE Transactions on Cybernetics, vol. 43, no. 2, pp. 673-684, April 2013.

[16] Y. Su, P. C. Muller and C. Zheng, "Global Asymptotic Saturated PID Control for Robot Manipulators," IEEE Transactions on Control Systems Technology, vol. 18, no. 6, pp. 1280-1288, Nov. 2010.

[17] M. Wang, A. Yang, "Dynamic Learning From Adaptive Neural Control of Robot Manipulators With Prescribed Performance," IEEE 
Transactions on Systems, Man, and Cybernetics: Systems, vol. PP no.99, pp.1-12, doi: 10.1109/TSMC.2016.2645942.

[18] A. Tayebi, S. Abdul, M. B. Zaremba and Y. Ye, "Robust Iterative Learning Control Design: Application to a Robot Manipulator," IEEE/ASME Transactions on Mechatronics, vol. 13, no. 5, pp. 608-613, Oct. 2008.

[19] T. D. Sanger, "Neural network learning control of robot manipulators using gradually increasing task difficulty," IEEE Transactions on Robotics and Automation, vol. 10, no. 3, pp. 323-333, Jun 1994.

[20] K. Fujimoto and T. Sugie, "Iterative learning control of Hamiltonian systems: I/O based optimal control approach," IEEE Transactions on Automatic Control, vol. 48, no. 10, pp. 1756-1761, Oct. 2003.

[21] Feng Lin and R. D. Brandt, "An optimal control approach to robust control of robot manipulators," IEEE Transactions on Robotics and Automation, vol. 14, no. 1, pp. 69-77, Feb 1998.

[22] V. Duchaine, S. Bouchard and C. M. Gosselin, "Computationally Efficient Predictive Robot Control," IEEE/ASME Transactions on Mechatronics, vol. 12, no. 5, pp. 570-578, Oct. 2007.

[23] M. Jin, S. H. Kang, P. H. Chang, J. Lee, "Robust Control of Robot Manipulators Using Inclusive and Enhanced Time Delay Control," IEEE/ASME Transactions on Mechatronics, vol.PP, no.99, pp.1-1 doi: 10.1109/TMECH.2017.2718108.

[24] A. Ferrara and G. P. Incremona, "Design of an Integral Suboptimal Second-Order Sliding Mode Controller for the Robust Motion Control of Robot Manipulators," IEEE Transactions on Control Systems Technology, vol. 23, no. 6, pp. 2316-2325, Nov. 2015.

[25] S. Islam and X. P. Liu, "Robust Sliding Mode Control for Robot Manipulators," IEEE Transactions on Industrial Electronics, vol. 58, no. 6, pp. 2444-2453, June 2011

[26] V. Utkin, Sliding Modes on control and Optimization, Springer Verlag, Berlin, Germany, 1992.

[27] Y. W. Liang, S. D. Xu, D. C. Liaw and C. C. Chen, "A Study of T-S Model-Based SMC Scheme With Application to Robot Control," IEEE Transactions on Industrial Electronics, vol. 55, no. 11, pp. 3964-3971, Nov. 2008.

[28] Q. Meng, T. Zhang, X. Gao and J. Y. Song, "Adaptive Sliding Mode Fault-Tolerant Control of the Uncertain Stewart Platform Based on Offline Multibody Dynamics," IEEE/ASME Transactions on Mechatronics, vol. 19, no. 3, pp. 882-894, June 2014.

[29] B. Xiao, Q. Hu and Y. Zhang, "Adaptive Sliding Mode Fault Tolerant Attitude Tracking Control for Flexible Spacecraft Under Actuator Saturation," IEEE Transactions on Control Systems Technology, vol 20 , no. 6 , pp. $1605-1612$, Nov. 2012

[30] H. Wang et al., "Design and Implementation of Adaptive Terminal Sliding-Mode Control on a Steer-by-Wire Equipped Road Vehicle," IEEE Transactions on Industrial Electronics, vol. 63, no. 9, pp. $5774-$ 5785 , Sept. 2016.

[31] G. Chen, Y. Song; Y. Guan, "Terminal Sliding Mode-Based Consensus Tracking Control for Networked Uncertain Mechanical Systems on Digraphs," IEEE Transactions on Neural Networks and Learning Systems, vol.PP, no.99, pp.1-8, doi: 10.1109/TNNLS.2016.2636323.

[32] Q. Xu, "Piezoelectric Nanopositioning Control Using Second-Order Discrete-Time Terminal Sliding-Mode Strategy," IEEE Transactions on Industrial Electronics, vol. 62, no. 12, pp. 7738-7748, Dec. 2015.

[33] T. Madani, B. Daachi and K. Djouani, "Modular-Controller-DesignBased Fast Terminal Sliding Mode for Articulated Exoskeleton Systems," IEEE Transactions on Control Systems Technology, vol. 25 no. 3, pp. 1133-1140, May 2017.

[34] C. U. Solis, J. B. Clempner and A. S. Poznyak, "Fast Terminal SlidingMode Control With an Integral Filter Applied to a Van Der Po Oscillator," IEEE Transactions on Industrial Electronics, vol. 64, no. 7, pp. 5622-5628, July 2017

[35] J. Zheng; H. Wang; Z. Man; J. Jin; M. Fu, "Robust Motion Control of a Linear Motor Positioner Using Fast Nonsingular Terminal Sliding Mode," IEEE/ASME Transactions on Mechatronics, vol.20, no.4, pp.1743-1752, Aug. 2015.

[36] C. K. Lin, "Nonsingular Terminal Sliding Mode Control of Robot Manipulators Using Fuzzy Wavelet Networks," IEEE Transactions on Fuzzy Systems, vol. 14, no. 6, pp. 849-859, Dec. 2006.

[37] Y. Wang, L. Gu, Y. Xu and X. Cao, "Practical Tracking Control of Robot Manipulators With Continuous Fractional-Order Nonsingular
Terminal Sliding Mode," IEEE Transactions on Industrial Electronics, vol. 63 , no. 10 , pp. 6194-6204, Oct. 2016.

[38] M. Jin, J. Lee and K. K. Ahn, "Continuous Nonsingular Terminal Sliding-Mode Control of Shape Memory Alloy Actuators Using Time Delay Estimation," IEEE/ASME Transactions on Mechatronics, vol. 20, no. 2, pp. 899-909, April 2015.

[39] F. J. Lin, P. H. Chou, C. S. Chen and Y. S. Lin, "Three-Degree-ofFreedom Dynamic Model-Based Intelligent Nonsingular Terminal Sliding Mode Control for a Gantry Position Stage," IEEE Transactions on Fuzzy Systems, vol. 20, no. 5, pp. 971-985, Oct. 2012

[40] S. Y. Chen and F. J. Lin, "Robust Nonsingular Terminal Sliding-Mode Control for Nonlinear Magnetic Bearing System," IEEE Transactions on Control Systems Technology, vol. 19, no. 3, pp. 636-643, May 2011.

[41] S. S. D. Xu, C. C. Chen and Z. L. Wu, "Study of Nonsingular Fast Terminal Sliding-Mode Fault-Tolerant Control," IEEE Transactions on Industrial Electronics, vol. 62, no. 6, pp. 3906-3913, June 2015

[42] M. Van, S. S. Ge and H. Ren, "Finite Time Fault Tolerant Control for Robot Manipulators Using Time Delay Estimation and Continuous Nonsingular Fast Terminal Sliding Mode Control," IEEE Transactions on Cybernetics, vol. 47, no. 7, pp. 1681-1693, July 2017.

[43] J. Zheng; H. Wang; Z. Man; J. Jin; M. Fu, "Robust Motion Control of a Linear Motor Positioner Using Fast Nonsingular Terminal Sliding Mode," IEEE/ASME Transactions on Mechatronics, vol.20, no.4, pp.1743-1752, Aug. 2015.

[44] J. Lee, P. H. Chang and M. Jin, "Adaptive Integral Sliding Mode Control With Time-Delay Estimation for Robot Manipulators," IEEE Transactions on Industrial Electronics, vol. 64, no. 8, pp. 6796-6804, Aug. 2017.

[45] R. Cui, L. Chen, C. Yang and M. Chen, "Extended State ObserverBased Integral Sliding Mode Control for an Underwater Robot With Unknown Disturbances and Uncertain Nonlinearities," IEEE Transactions on Industrial Electronics, vol. 64, no. 8, pp. 6785-6795, Aug. 2017.

[46] V. Parra-Vega, S. Arimoto, Yun-Hui Liu, G. Hirzinger and P. Akella, "Dynamic sliding PID control for tracking of robot manipulators: theory and experiments," IEEE Transactions on Robotics and Automation, vol. 19 , no. 6, pp. 967-976, Dec. 2003

[47] G. P. Incremona, A. Ferrara and L. Magni, "MPC for Robot Manipulators With Integral Sliding Modes Generation," IEEE/ASME Transactions on Mechatronics, vol. 22, no. 3, pp. 1299-1307, June 2017.

[48] M. Van, S. S. Ge and H. Ren, "Robust Fault-Tolerant Control for a Class of Second-Order Nonlinear Systems Using an Adaptive ThirdOrder Sliding Mode Control," IEEE Transactions on Systems, Man, and Cybernetics: Systems, vol. 47, no. 2, pp. 221-228, Feb. 2017.

[49] Q. Xu, "Continuous Integral Terminal Third-Order Sliding Mode Motion Control for Piezoelectric Nanopositioning System," IEEE/ASME Transactions on Mechatronics, vol.PP, no.99, pp.1-1 doi: 10.1109/TMECH.2017.2701417.

[50] Q. Xu, "Digital Integral Terminal Sliding Mode Predictive Control of Piezoelectric-Driven Motion System," IEEE Transactions on Industrial Electronics, vol. 63, no. 6, pp. 3976-3984, June 2016.

[51] S. Yin and B. Xiao, "Tracking Control of Surface Ships With Disturbance and Uncertainties Rejection Capability," IEEE/ASME Transactions on Mechatronics, vol. 22, no. 3, pp. 1154-1162, June 2017.

[52] G. P. Incremona, M. Rubagotti and A. Ferrara, "Sliding Mode Control of Constrained Nonlinear Systems," IEEE Transactions on Automatic Control, vol. 62, no. 6, pp. 2965-2972, June 2017.

[53] Y. W. Liang, S. D. Xu, D. C. Liaw and C. C. Chen, "A Study of T-S Model-Based SMC Scheme With Application to Robot Control," IEEE Transactions on Industrial Electronics, vol. 55, no. 11, pp. 3964-3971, Nov. 2008.

[54] J. Zhang, X. Liu, Y. Xia, Z. Zuo and Y. Wang, "Disturbance ObserverBased Integral Sliding-Mode Control for Systems With Mismatched Disturbances," IEEE Transactions on Industrial Electronics, vol. 63, no. 11, pp. 7040-7048, Nov. 2016.

[55] Y. Cao and X. B. Chen, "Disturbance-Observer-Based Sliding-Mode Control for a 3-DOF Nanopositioning Stage," IEEE/ASME Transactions on Mechatronics, vol. 19, no. 3, pp. 924-931, June 2014.

[56] N. M. Dehkordi, N. Sadati and M. Hamzeh, "A Robust Backstepping High-Order Sliding Mode Control Strategy for Grid-Connected DG 
Units With Harmonic/Interharmonic Current Compensation Capability," IEEE Transactions on Sustainable Energy, vol. 8, no. 2, pp. 561-572, April 2017.

[57] A. Levant, "Robust exact differentiation via sliding mode technique," Automatica, vol. 34, no. 3, pp. 379-384, 1998.

[58] M. Van, H. J. Kang, Y. S. Suh, "Second order sliding mode based output feedback tracking control for uncertain robot manipulators," International Journal of Advanced Robotic Systems, vol. 10, no. 1, pp. 1-9, 2013.

[59] M. Van, H. J. Kang, K. S. Shin, "Backstepping quasi-continuous highorder sliding mode control for a T-S fuzzy system with an application for a two-link robot control," Proceeding of the institution of Mechanical Engineers, Part C: Journal of Mechanical Engineering, vol. 228, no. 6, pp. 1488-1500, 2014.

[60] M. Van, H. J. Kang, "Robust fault tolerant control for uncertain robot manipulators based on adaptive quasi-continuous high-order sliding mode and neural network," Proceeding of the institution of Mechanical Engineers, Part C: Journal of Mechanical Engineering, vol. 229, no. 8 , pp. 1425-1446, 2015.

[61] S. Tong, B. Huo and Y. Li, "Observer-Based Adaptive Decentralized Fuzzy Fault-Tolerant Control of Nonlinear Large-Scale Systems With Actuator Failures," IEEE Transactions on Fuzzy Systems, vol. 22, no. 1, pp. 1-15, Feb. 2014

[62] S. Tong, T. Wang and Y. Li, "Fuzzy Adaptive Actuator Failure Compensation Control of Uncertain Stochastic Nonlinear Systems With Unmodeled Dynamics," IEEE Transactions on Fuzzy Systems, vol. 22, no. 3, pp. 563-574, June 2014

[63] S. Tong, L. Zhang and Y. Li, "Observed-Based Adaptive Fuzzy Decentralized Tracking Control for Switched Uncertain Nonlinear Large-Scale Systems With Dead Zones," IEEE Transactions on Systems, Man, and Cybernetics: Systems, vol. 46, no. 1, pp. 37-47, Jan. 2016.

[64] S. Tong, Y. Li and S. Sui, "Adaptive Fuzzy Tracking Control Design for SISO Uncertain Nonstrict Feedback Nonlinear Systems," IEEE Transactions on Fuzzy Systems, vol. 24, no. 6, pp. 1441-1454, Dec. 2016.

[65] Q. Shen, B. Jiang, P. Shi and C. C. Lim, "Novel Neural Networks-Based Fault Tolerant Control Scheme With Fault Alarm," IEEE Transactions on Cybernetics, vol. 44, no. 11, pp. 2190-2201, Nov. 2014.

[66] B. Huo, Y. Xia, L. Yin and M. Fu, "Fuzzy Adaptive Fault-Tolerant Output Feedback Attitude-Tracking Control of Rigid Spacecraft," IEEE Transactions on Systems, Man, and Cybernetics: Systems, vol. 47, no. 8, pp. 1898-1908, Aug. 2017.

[67] L. Li, S. X. Ding, J. Qiu and Y. Yang, "Real-Time Fault Detection Approach for Nonlinear Systems and its Asynchronous T-S Fuzzy Observer-Based Implementation," IEEE Transactions on Cybernetics, vol. 47, no. 2, pp. 283-294, Feb. 2017.

[68] M. Biglarbegian, W. W. Melek and J. M. Mendel, "Design of Novel Interval Type-2 Fuzzy Controllers for Modular and Reconfigurable Robots: Theory and Experiments," IEEE Transactions on Industrial Electronics, vol. 58, no. 4, pp. 1371-1384, April 2011.

[69] Y. X. Li and G. H. Yang, "Robust Adaptive Fault-Tolerant Control for a Class of Uncertain Nonlinear Time Delay Systems," IEEE Transactions on Systems, Man, and Cybernetics: Systems, vol. 47, no. 7, pp. 15541563, July 2017.

[70] J. X. Zhang; G. H. Yang, "Prescribed Performance Fault-Tolerant Control of Uncertain Nonlinear Systems With Unknown Control Directions," IEEE Transactions on Automatic Control, vol.PP, no.99, pp.1-1, doi: 10.1109/TAC.2017.2705033.

[71] L. Y. Hao; J. H. Park; D. Ye, "Integral Sliding Mode Fault-Tolerant Control for Uncertain Linear Systems Over Networks With Signals Quantization," IEEE Transactions on Neural Networks and Learning Systems, vol.PP, no.99, pp.1-13 doi: 10.1109/TNNLS.2016.2574905.

[72] A. Brian, O. Khatib, and J. Burdick, "The explicit dynamic model and inertial parameters of the PUMA560 arm," in Proc. IEEE Int. Conf. Robot. Autom., vol. 3. San Francisco, CA, USA, 1986, pp. 510-518.

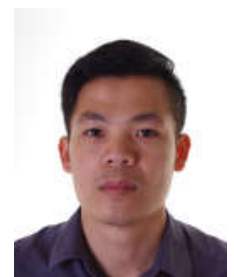

Mien Van received his B.S. degree in Electrical Engineering from Danang University of Technology, DaNang city, Vietnam, in 2009. He received his Ph.D. degree in the School of Electrical Engineering, University of Ulsan, South Korea in 2015.

He was a Post-Doctoral Research Fellow with the Advanced Robotics Centre, Faculty of Engineering, National University of Singapore, Singapore and the WMG, University of Warwick, UK, and an Independent Research Fellow/Lecturer with the Nottingham Trent University, UK from 2015. From 2018, He is a Senior Research Fellow with the College of Engineering, Mathematics and Physical Sciences, University of Exeter, UK. He is a recipient of the IET Science, Measurement \& Technology Premium Awards in 2017. His research interests include Assistive robotics, Robot control, Robot-vision system, Fault diagnosis and fault tolerant, Machine learning, and Sensing and Perception.

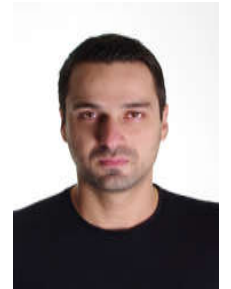

Michalis Mavrovouniotis (M'13) received the B.Sc. degree in Computer Science from the University of Leicester, Leicester, U.K., in 2008, the M.Sc. degree in Natural Computation from the University of Birmingham, Birmingham, U.K., in 2009, and the Ph.D degree in Computer Science from the University of Leicester, in 2013. He is a Lecturer with the Nottingham Trent University, Nottingham, U.K

His current research interests include evolutionary computation, swarm intelligence, memetic computing, combinatorial optimization problems, computational intelligence in dynamic and uncertain environments, and relevant real-world applications.

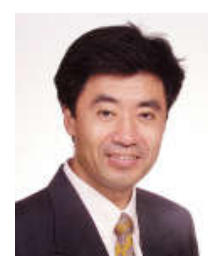

Shuzhi Sam Ge (S'90-M'92-SM'00-F'06) received the B.Sc. degree from the Beijing University of Aeronautics and Astronautics, Beijing, China, in 1986, and the Ph.D. degree from the Imperial College of Science, Technology and Medicine, University of London, London, U.K., in 1993.

$\mathrm{He}$ is the Director of Social Robotics Laboratory, Interactive Digital Media Institute, and the Professor with the Department of Electrical and Computer Engineering, National University of Singapore, Singapore. He has co-authored seven books and over 300 international journal and conference papers. His current research interests include social robotics, adaptive control, intelligent systems, and artificial intelligence. Prof. Ge is an Editor-in-Chief of the International Journal of Social Robotics, Springer. He has served/been serving as an Associate Editor for a number of flagship journals, including the IEEE TRANSACTIONS ON AUTOMATIC CONTROL, the IEEE TRANSACTIONS ON CONTROL SYSTEMS TECHNOLOGY, the IEEE TRANSACTIONS ON NEURAL NETWORKS, and Automatica. He also serves as a Book Editor of the Taylor \& Francis Automation and Control Engineering Series. At the IEEE Control Systems Society, he served/serves as the Vice President for Technical Activities, from 2009 to 2010, the Vice President of Membership Activities, from 2011 to 2012, a Member of Board of Governors of the IEEE Control Systems Society, from 2007 to 2009. He is a fellow of the International Federation of Automatic Control, the Institute of Engineering and Technology, and Singapore Association Engineering. 